\title{
Complicated variations in the early optical afterglow of GRB 090726
}

\author{
V. Šimon ${ }^{1}$, C. Polášek ${ }^{1}$, M. Jelínek ${ }^{2}$, R. Hudec ${ }^{1}$, and J. Štrobl ${ }^{1}$ \\ 1 Astronomical Institute, Academy of Sciences of the Czech Republic, 25165 Ondřejov, Czech Republic \\ e-mail: simon@asu.cas.cz \\ 2 Instituto de Astrofísica de Andalucía CSIC, Apartado de Correos, 3.004, 18.080 Granada, Spain
}

Received 1 October 2009 / Accepted 4 November 2009

\section{ABSTRACT}

\begin{abstract}
Aims. We report on the detection of an early rising phase of optical afterglow (OA) of a long GRB 090726. We resolve a complicated profile of the optical light curve. We also investigate the relation of the optical and X-ray emission of this event.

Methods. We made use of the optical photometry of this OA obtained by the $0.5 \mathrm{~m}$ telescope of AI AS CR, supplemented by the data obtained by other observers, and the X-ray Swift/XRT data.

Results. The optical emission peaked at $\sim 17.5 \mathrm{mag}(R)$ at $t-T_{0} \approx 500 \mathrm{~s}$. We find a complex profile of the light curve during the early phase of this OA: an approximately power-law rise, a rapid transition to a plateau, a weak flare superimposed on the center of this plateau, and a slowly steepening early decline followed by a power-law decay. We discuss several possibilities for explaining the short flare on the flat top of the optical light curve at $t-T_{0} \approx 500 \mathrm{~s}$. Activity of the central engine is favored, although reverse shock cannot be ruled out. We show that power-law outflow with $\Theta_{\text {obs }} / \Theta_{c}>2.5$ is the best case for the OA of GRB 090726. The initial Lorentz factor is $\Gamma_{0} \approx 230-530$ in the case of propagation of the blast wave in a homogeneous medium, while propagation of this wave in a wind environment gives $\Gamma_{0} \approx 80-300$. The value of $\Gamma_{0}$ in GRB 090726 thus falls into the lower half of the range observed in GRBs and it may even lie on the lower end. We also show that both the optical and X-ray emission decayed simultaneously and that the spectral profile from X-ray to the optical band did not vary. This is true for both the time periods before and after the break in the $\mathrm{X}$-ray light curve. This break can be regarded as achromatic. The available data show that neither the dust nor the gaseous component of the circumburst medium underwent any evolution during the decay of this OA, that is, after $t-T_{0}<3000 \mathrm{~s}$. We also show that this OA belongs to the least luminous ones in the phase of its power-law decay, corresponding to what is observed for the ensemble of OAs of long GRBs.
\end{abstract}

Key words. stars: gamma-ray burst: individual: GRB 090726 - radiation mechanisms: non-thermal - plasmas - galaxies: ISM ISM: jets and outflows - galaxies: starburst

\section{Introduction}

GRB 090726 was a long gamma-ray burst (GRB) localized by Swift/BAT on 26 July 2009 at 22:42:27.8 UT. It displayed a single-peak profile, with $T_{90}(15-350 \mathrm{keV})=67 \pm 15 \mathrm{~s}$. Fluence in the $15-150 \mathrm{keV}$ band was $(8.6 \pm 1.0) \times 10^{-7} \mathrm{erg} \mathrm{cm}^{-2} \mathrm{~s}^{-1}$. The 1 -s peak flux was $0.7 \pm 0.2$ photon $\mathrm{cm}^{-2} \mathrm{~s}^{-1}$ (Page et al. 2009).

The GRB coordinates were available via GCN within $65 \mathrm{~s}$, although Swift could not follow-up until $\sim 3.6 \mathrm{ks}$ after the trigger because of an Earth-limb constraint. Redshift of this GRB is $z=$ 2.71, as determined from the lines in optical spectrum of the optical afterlow (OA) (Fatkhullin et al. 2009).

We report the early observations of the OA of this GRB performed by the robotic $0.5 \mathrm{~m}$ telescope (D50) of the Astronomical Institute of AS CR in Ondřejov, Czech Republic. The first image of the D50 started at 22:45:41.0 UT, i.e. $194 \mathrm{~s}$ after the trigger. The telescope took images until 23:35:35 UT (for 50 min), until a pointing limit was reached. On all of the 107 unfiltered, $20 \mathrm{~s}$ exposures, the OA can be detected, although we co-added some of the images to improve the signal-to-noise ratio.

\section{Observations and data analysis}

D50 is a robotic, 500/1975 mm Newtonian telescope, located at AI AS CR in Ondřejov. It is equipped with a CCD camera FLI IMG 4710 (CCD chip E2V 47-10, mid-band coating, $1024 \times 1024$ pixels), focuser FLI DF-2, and a field corrector
TeleVue Paracorr PSB-11000. It is controlled by RTS2 software (Kubánek et al. 2006) and makes use of astrometric code by Lang et al. (2009). The field of view is $20 \times 20$ arcmin. To reach deeper magnitudes, unfiltered observations were carried out. Their peak sensitivity is close to the $R$ filter. An exposure time of $20 \mathrm{~s}$ was used for each CCD image.

Aperture photometry using phot routine within IRAF/DAOPhot ${ }^{1}$ was used on the OA and 8 comparison stars. The reference grid of these 8 stars was then calibrated with brightnesses from USNO-A2.0 (Monet et al. 1998) using robust fitting algorithms to avoid any possible influence by star variability. The values we obtained for brightnesses of the calibration stars are in Table 1 . Denoted error estimates $(1 \sigma)$ are for relative brightnesses within this frame, external error binding to USNO system ( $\sim 0.04 \mathrm{mag})$ and error of the USNO calibration itself $(\sim 0.2 \mathrm{mag})-$ are much larger than this. As we have no afterglow color information, we cannot transform the CR (clear, $R$-calibrated) magnitudes into real $R$ band. Because the airmass changed only between 1.180 and 1.124 during the observation, the atmospheric color effects should be negligible. The CR magnitude of the OA only differs from the $R$ band by the difference between the OA color and the mean color of the

\footnotetext{
IRAF is distributed by the National Optical Astronomy Observatory, which is operated by the Association of Universities for Research in Astronomy, Inc., under co-operative agreement with the National Science Foundation.
} 
Table 1. Calibration stars used, together with their USNO-A2.0 derived magnitudes.

\begin{tabular}{lccc}
\hline \hline id & mag & id & mag \\
\hline 1 & $14.203 \pm 0.001$ & 5 & $15.854 \pm 0.006$ \\
2 & $16.602 \pm 0.010$ & 6 & $15.980 \pm 0.006$ \\
3 & $16.689 \pm 0.011$ & 7 & $17.139 \pm 0.015$ \\
4 & $16.012 \pm 0.007$ & 8 & $17.185 \pm 0.018$ \\
\hline
\end{tabular}

Table 2. Observing log of Ondřejov data.

\begin{tabular}{cccllc}
\hline \hline$t-T_{0}$ & $E$ & $\mathrm{mag}$ & $t-T_{0}$ & $E$ & $\mathrm{mag}$ \\
\hline 204.2 & 20 & $18.12 \pm 0.12$ & 741.3 & 77 & $17.79 \pm 0.04$ \\
232.2 & 20 & $17.97 \pm 0.11$ & 825.5 & 76 & $17.79 \pm 0.04$ \\
260.7 & 20 & $17.85 \pm 0.10$ & 910.5 & 76 & $17.82 \pm 0.05$ \\
288.7 & 20 & $17.76 \pm 0.08$ & 995.5 & 77 & $17.93 \pm 0.05$ \\
317.2 & 20 & $17.77 \pm 0.09$ & 1123.0 & 162 & $17.94 \pm 0.04$ \\
345.2 & 20 & $17.82 \pm 0.09$ & 1292.2 & 161 & $17.91 \pm 0.03$ \\
373.8 & 20 & $17.76 \pm 0.08$ & 1462.1 & 162 & $18.06 \pm 0.04$ \\
430.3 & 20 & $17.75 \pm 0.09$ & 1631.1 & 161 & $18.12 \pm 0.04$ \\
458.4 & 20 & $17.67 \pm 0.07$ & 1843.1 & 246 & $18.20 \pm 0.04$ \\
486.8 & 20 & $17.60 \pm 0.08$ & 2097.6 & 247 & $18.34 \pm 0.04$ \\
514.8 & 20 & $17.55 \pm 0.07$ & 2351.7 & 246 & $18.42 \pm 0.05$ \\
557.3 & 48 & $17.65 \pm 0.05$ & 2648.2 & 331 & $18.66 \pm 0.04$ \\
613.8 & 48 & $17.83 \pm 0.06$ & 3015.1 & 387 & $18.70 \pm 0.05$ \\
670.3 & 48 & $17.75 \pm 0.05$ & & & \\
\hline
\end{tabular}

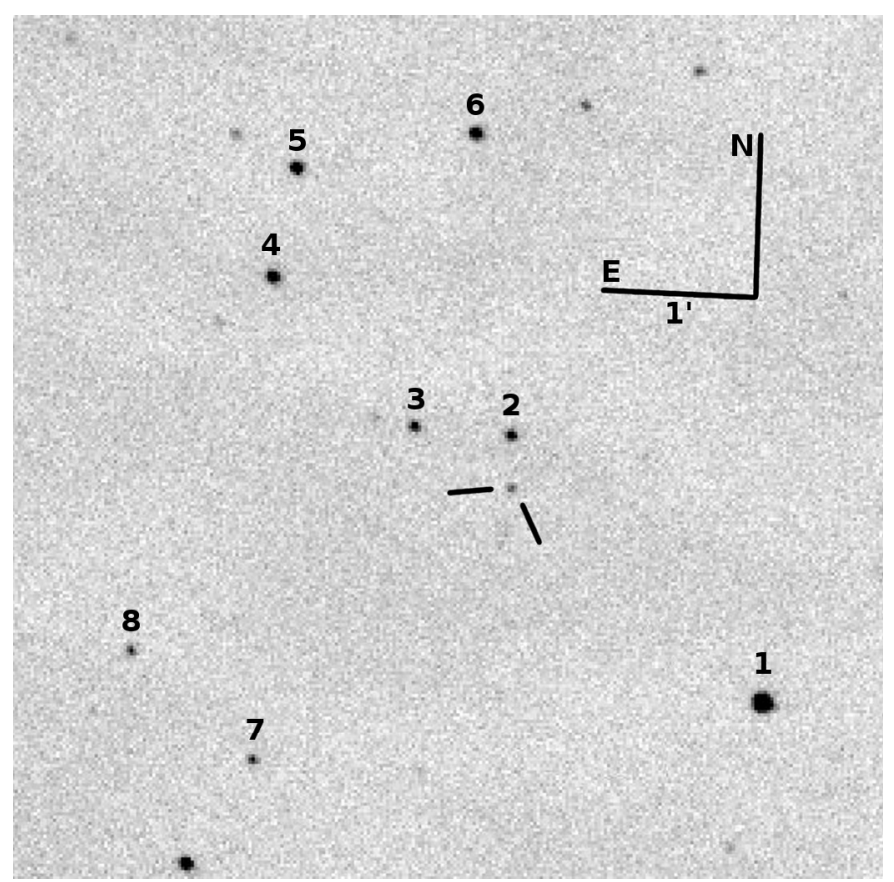

Fig. 1. CCD image of OA of GRB 090726 secured with the D50 $\mathrm{cm}$ telescope. It was obtained close to the time of the peak brightness of this OA, at $t-T_{0} \approx 670 \mathrm{~s}$. Only a part of the original image is shown. The OA and the comparison stars are marked. See Sect. 2 for details.

calibration stars. If we assume the (typically) constant color of the OA, this difference also stays constant. The data are given in Table 2. Time elapsed from the GRB trigger in seconds is marked as $t-T_{0}$, while $E$ refers to the exposure time in seconds (some images are co-added). Magnitude is denoted as mag, including its $1 \sigma$ error.

An example of our CCD image of the OA, obtained at $t-T_{0} \approx$ $670 \mathrm{~s}$, which is near its peak magnitude, is displayed in Fig. 1.
Only a part of the original $20 \times 20$ arcmin CCD image is shown. The OA is clearly defined as a new, variable source.

The resulting light curve of the OA is displayed in Fig. 2. The $R$ band observations of Moskvitin et al. (2009) and Volnova et al. (2009) were also included. The data by Moskvitin et al. (2009) fit our unfiltered observations nicely. The optical transient was observed with a $60 \mathrm{~cm}$ telescope at Crni Vrh (Maticic \& Skvarc 2009) simultaneously with our measurements. We found that their light curve shows a large scatter. The main contribution of their data is that their first point was obtained before the start of our observations $\left(t-T_{0}=154 \mathrm{~s}\right.$ vs. $\left.t-T_{0}=194 \mathrm{~s}\right)$. The brightness was fainter than or comparable to our first observation, which helps constrain any possible large-amplitude initial flash. To resolve the profile of the early phase of OA in detail, both the panels with a linear and log-log scales of the axes are shown in Fig. 2.

To show the profile of the early phase of OA more clearly and to lower the scatter of the observations, Ondřejov data were fitted by the code HEC13 written by Harmanec (1992). The code is based on the method of Vondrák $(1969,1977)$, who improved the original method of Whittaker (Whittaker \& Robinson 1946). A full description of the method can be found in Vondrák (1969). This method can fit a smooth curve to the data regardless of their profile. These data can be distributed in time non-uniformly. The method is based on minimizing the value $Q=F+\lambda^{2} S$, where $F=\sum p\left(y_{\mathrm{i}}-y_{\mathrm{i}}^{\prime}\right)^{2}$ denotes the degree of smoothing $(y$ being the smoothed and $y^{\prime}$ the observed value of the variable), $S=\sum\left(\Delta^{3} y_{\mathrm{i}}\right)^{2}$ is the measure of roughness of the curve, $\lambda^{2}$ is a constant to be selected, and it defines how much the curve will be smoothed. HEC13 makes use of two input parameters, $\epsilon$ (in dimensionless units) and $\Delta T$. The quantity $\epsilon=1 / \lambda^{2}$ determines how "tight" the fit will be, that is, if only the main profile or also the high-frequency variations are to be reproduced. The quantity $\Delta T$ is the interval over which the data are binned before smoothing. The resulting fit consists of the mean points, calculated to the individual observed points of the curve. A set of fits to the data with the different input parameters $\epsilon$ and $\Delta T$ was generated and submitted for inspection. Only the fit to the plateau and the decay are shown in Fig. 2, because the transition between the rising branch and the plateau proved too sharp to give any reliable fit. Also the narrow flare at $t-T_{0} \approx 500 \mathrm{~s}$ was omitted from the fitting. It was found that the fit with $\epsilon=1, \Delta T=400 \mathrm{~s}$ reproduces the main features of the light curve and significantly enhances the visibility of the main profile. The residuals of this fit are displayed in Fig. 2c, and show that four points belonging to the flare and not included in the fit, clearly deviate from the profile of this curve. This fit also allows us to conclude that no other flare with the amplitude larger than the errors of the data occurred during Ondřejov observations. It is true that this method is somewhat subjective, but it enables us to find a compromise between a curve running through all the observed values and an ideal smooth curve. We preferred to use this method because it does not make any assumptions about the profile of the fitted data.

The relation of the optical and X-ray flux and the evolution of the X-ray hardness ratio were investigated using the Swift/XRT (Evans et al. 2007, 2009) data of the afterglow of GRB 090726. Only the $R$ band data of Moskvitin et al. (2009) were used, because Ondřejov observations must have already finished. In order to get an analogy to the color index, the quantity $R X=$ $\log \left[F_{v}(R)\right]-\log \left[I_{\mathrm{X}} / v\right]$ was calculated, where $F_{v}(R)$ is the flux in the $R$ band, $I_{\mathrm{X}}$ the observed $\mathrm{X}$-ray flux in the $0.3-10 \mathrm{keV}$ band, and $v$ the frequency. The conversion between the $R$ band magnitude and flux was carried out according to the relation of 

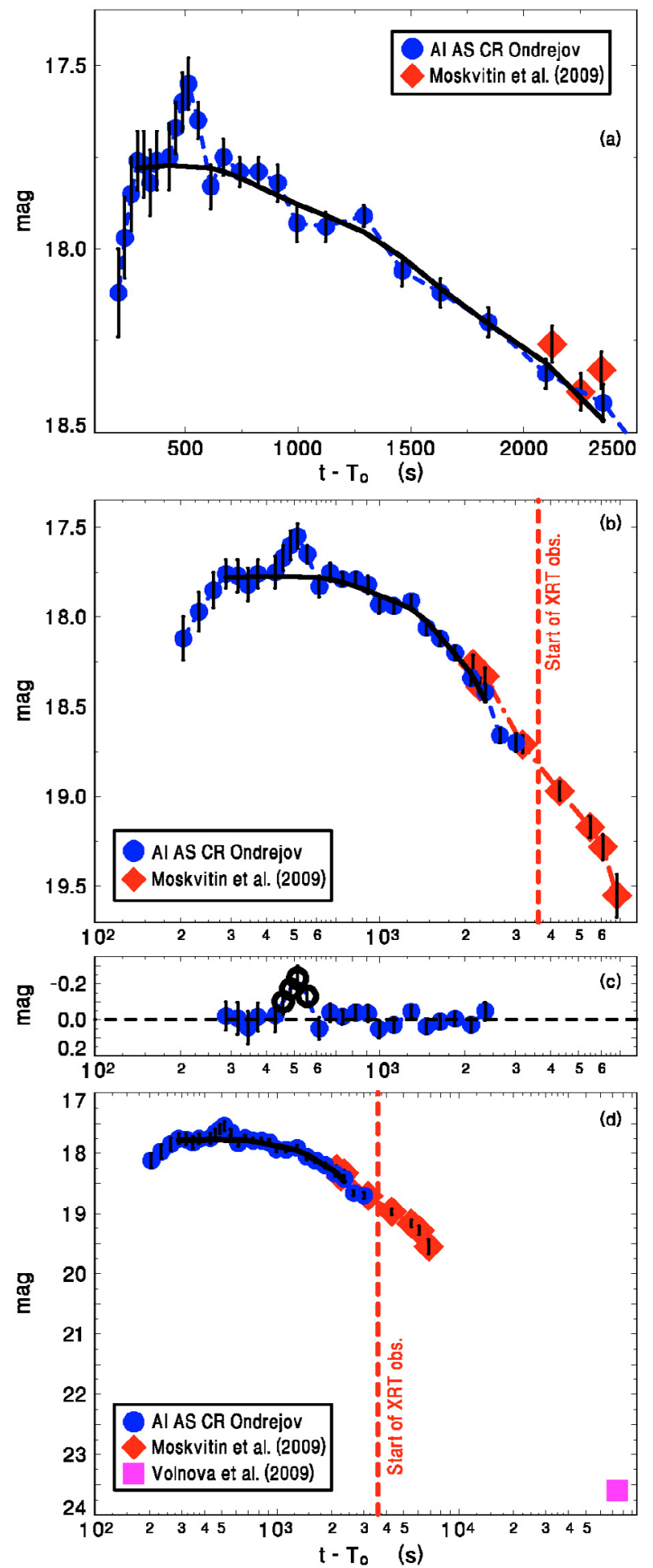

Fig. 2. Light curve of OA of GRB 090726 in the red spectral region. The data coming from the individual observers are resolved. HEC13 fit to Ondřejov data is marked by the smooth, solid line. a) The very early phase plotted with a linear scale on the abscissa. b) The early phase with a logarithmic scale of the abscissa. c) Residuals of HEC13 fit. The open circles are highlighting the flare. d) The first day of OA. A vertical line denotes the start of Swift/XRT observations. The error bars represent the uncertainties at the $1 \sigma$ level. See Sect. 2 for details.

Clark et al. (2000). The value of $v=4 \times 10^{17} \mathrm{~Hz}$ was used for $I_{\mathrm{X}}$. Also, hardness $H$ of the $\mathrm{X}$-ray spectrum was determined from the count rates $\mathrm{cr}$ in the hard and soft bands as follows: $H=\log \left(c r_{\text {hard }}(1.5-10 \mathrm{keV})\right)-\log \left(c r_{\text {soft }}(0.3-1.5 \mathrm{keV})\right)$. The
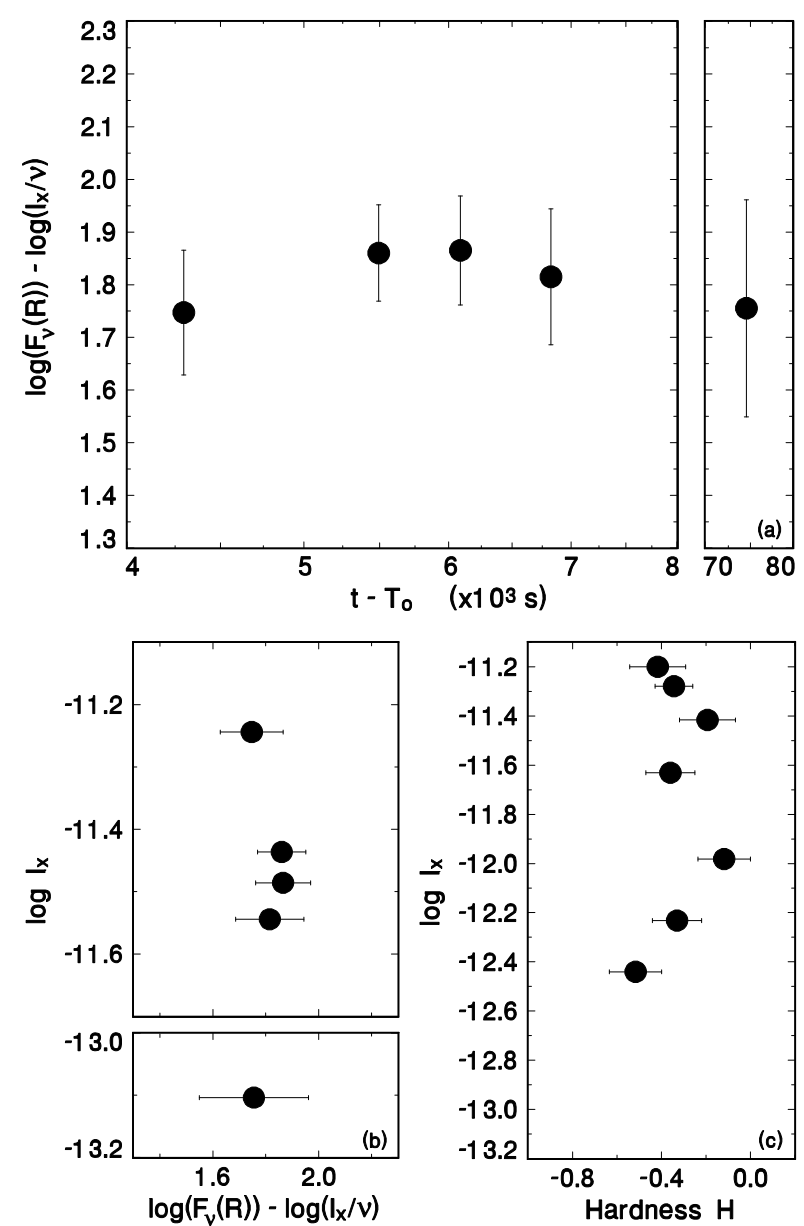

Fig. 3. a) Time evolution of the relation of the optical and X-ray flux $(0.3-10 \mathrm{keV})$ in the OA of GRB 090726, defined in the text. b) Evolution of this relation with the X-ray flux. c) Evolution of X-ray hardness $H$ with the X-ray flux. The optical and X-ray fluxes of the OA decline in all three diagrams. The error bars were calculated from the uncertainties given on both the optical and X-ray data at the 90 percent level. See Sect. 2 for details.

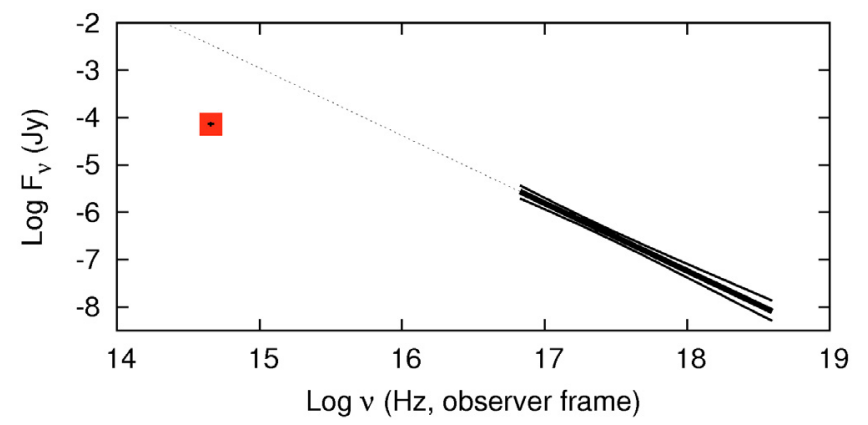

Fig. 4. SED of the afterglow of GRB 090726 at $t-T_{0}=4000 \mathrm{~s}$. The X-ray data come from Swift/XRT, and the optical measurement represents the extrapolation of Ondřejov data. See Sect. 2 for details.

results are displayed in Fig. 3. Both $R X$ and $H$ remain constant within the observational errors during the decay of the afterglow.

The spectral energy distribution (SED) of the afterglow of GRB 090726 is displayed in Fig. 4. The decay slope of Ondřejov data for $t-T_{0}>1730 \mathrm{~s}$ was extrapolated to $t-T_{0}=4000 \mathrm{~s}$, that is, to the time for which Swift/XRT observations exist. The $\mathrm{X}$-ray data were corrected for the absorption given by Page et al. (2009). The $R$ band magnitude was corrected for the Galactic 
reddening by $A_{R}=0.11$ mag according to the maps by Schlegel et al. (1998).

\section{Results}

We report on a detection of early, rising OA of GRB 090726. Its light curve displayed a complicated profile, which can be summarized as:

1) steep rise until $t-T_{0} \approx 300 \mathrm{~s}$, which finished with a rapid transition to a plateau lasting until $t-T_{0} \approx 800 \mathrm{~s}$,

2) a short flare on the flat top (plateau) of the optical light curve at $t-T_{0} \approx 500 \mathrm{~s}$,

3) only a slowly steepening decline in $800 \mathrm{~s}<t-T_{0}<1400 \mathrm{~s}$,

4) steepening power-law decay at $t-T_{0} \approx 1400 \mathrm{~s}$ lasting at least until $t-T_{0} \approx 7000 \mathrm{~s}$.

Another break in the decay occurred between $t-T_{0} \approx 7000 \mathrm{~s}$ and $t-T_{0} \approx 0.86 \mathrm{~d}$ (see Volnova et al. 2009).

The initial rise for $t-T_{0}<300 \mathrm{~s}$ (the first 4 points in Fig. 2) can be approximated by a power law with the index $\alpha_{\mathrm{r}}=0.94 \pm 0.05$. This small $\alpha_{\mathrm{r}}$ speaks in favor of propagation of the blast wave in a wind of the progenitor rather than in the interstellar medium. Since the observed part of the rise of OA occurred well after the end of the gamma-ray emission, this is a thin-shell expansion (e.g. Zhang \& Kobayashi 2005).

A short flare, superimposed on the flat top of the optical light curve, attained the peak magnitude at $t-T_{0} \approx 500 \mathrm{~s}$. A powerlaw fit yields $\alpha_{\mathrm{rF}}=2.7 \pm 0.8$ for the rising branch, while the decay can be fitted with $\alpha_{\mathrm{dF}}=-3.6 \pm 0.8$. This decay is considerably steeper than the later power-law one. Prompt emission of GRB is ruled out because the flare occurred well after the end of the observed GRB emission. Also, a density enhancement is less likely because the decline shallower than $t^{-(2+\beta)}$ is expected (Fenimore et al. 1996). Assuming a common spectral index $\beta=1$ (Nardini et al. 2006), the decline should be shallower than $t^{-3}$ in this case. If this flare is caused by reverse shock, then it sorts OA of GRB 090726 to type II, as defined by Zhang et al. (2003). However, the observed decay appears to be too steep because $\alpha_{\mathrm{dF}} \approx 2$ is expected (Kobayashi 2000), although a wind could help steepen this decline (Greiner et al. 2008). In this regard, we note that any additional flash, like the one in GRB 990123 (Akerlof et al. 1999) and caused by another reverse shock, could occur before the start of Ondřejov observations only if its peak magnitude were comparable to or fainter than that of the peak observed at $t-T_{0} \approx 500 \mathrm{~s}$. The absence of this flash is strengthened by the first point of Maticic \& Skvarc (2009) also showing that the OA was not brighter than in the subsequent Ondřejov data. The steep decay of the observed flare speaks in favor of activity by the central engine (Fan et al. 2008). This flare occurred during the phase in which OAs observed in X-rays often display a plateau and/or flares (e.g. Burrows et al. 2005; Willingale et al. 2007). A flare can appear if the central engine continues to create shells of much less power at late times with smaller $\Gamma$ even after GRB (Ghisellini et al. 2007).

The profile of OA of GRB 090726 near the peak magnitude can be used to distinguish between various profiles of the jet and the viewing angles, using the recent models by Panaitescu \& Vestrand (2008). The top of this OA is strikingly different from the sharp ones of GRB 060418 and GRB 060607A (Molinari et al. 2007; Nysewander et al. 2009). Its flat top speaks against isotropic outflow or a jet seen face-on. A jet with a sharp angular boundary with $\Theta_{\text {obs }} / \Theta_{\text {jet }}>2$ can give rise to a short plateau, but the time of its peak luminosity $t_{\text {peak }}$ occurs at $t-T_{0}>1000 \mathrm{~s}$ for $z=2.71$, which is too late to be consistent with our data. Power-law outflow with $\Theta_{\text {obs }} / \Theta_{c}>2.5\left(\Theta_{c}\right.$ being angular size of the core) is the best case for OA of GRB 090726. It yields a flat top at $t-T_{0}$ of several hundred seconds. The flat top thus can be caused by observing the power-law jet of the OA of GRB 090726 off-axis.

Some OAs were observed to display a re-brightening or a plateau between rest frame $\left(t-T_{0}\right)_{\text {rest }}$ of 0.01 and $0.02 \mathrm{~d}$ (i.e. $t-T_{0}$ between $\sim 3200$ and $\sim 6400 \mathrm{~s}$ for $z=2.71$ ) (Klotz et al. 2005). This time period corresponds to the phase of the powerlaw decay in OA of GRB 090726. Also, the X-ray 0.3-10 keV data display only smooth, power-law decay without any plateau in this epoch.

Determining the initial Lorentz factor $\Gamma_{0}$ of GRB requires a knowledge of its isotropic energy release $E_{\text {iso. Since only a }}$ 15-150 keV spectrum, best-fitted by a simple power-law model (Page et al. 2009), is available, we estimated $E_{\text {iso }}$ from the Amati relation (Amati et al. 2002). The observed spectrum suggests $E_{\text {iso }}>2 \times 10^{52} \mathrm{erg}$. The upper limit of GRBs is $E_{\text {iso }} \approx 3 \times 10^{54} \mathrm{erg}$. Following the approach of Molinari et al. (2007), the initial Lorentz factor of GRB 090726 turns out to be $\Gamma_{0} \approx 230-530$ in case of propagation of the blast wave in a homogeneous medium, while propagation of this wave in a wind environment gives $\Gamma_{0} \approx 80-300$. The density $n=1 \mathrm{~cm}^{-3}$ and the radiative efficiency $\eta=0.2$ were assumed in both cases. Taking $t_{\text {peak }}=300 \mathrm{~s}$ or $500 \mathrm{~s}$ has only a minor effect on $\Gamma_{0}$ in our case because the uncertainties in $E_{\text {iso }}$ dominate. Rykoff et al. (2009) find $\Gamma_{0}$ between $\sim 100$ and $\sim 1000$ in their ensemble of GRBs. The value of $\Gamma_{0}$ in GRB 090726 thus falls into the lower half of this range and, given the uncertainty in its $E_{\text {iso }}$, it may even lie at the lower end.

Ondřejov data show that the optical light curve achieves a power-law decay with $\alpha_{\mathrm{dO}}=-1.02 \pm 0.12$ from $t-T_{0}=1730 \mathrm{~s}$ up to the end of the series, which is a typical value for OAs. The X-ray observations of the afterglow of GRB 090726 started when the OA emission was already declining (see Fig. 2b), d)). The decline of the X-ray emission was a power law with $\alpha_{\mathrm{dX}}=-1.27 \pm 0.09$ before a break at $t-T_{0}=51_{-7}^{+18} \mathrm{ks}$ (Page et al. 2009). Both $\alpha_{\mathrm{dO}}$ and $\alpha_{\mathrm{dX}}$ can be regarded as identical at $\sim 2 \sigma$ level. The ratio of the optical and X-ray flux can be considered constant (Fig. 3). All this suggests that both the optical and X-ray emission decayed simultaneously and that the spectral profile from X-ray to the optical band did not vary. This is true for both the time period before and after the break in the decaying light curve, and no significant flares were observed in the data set of the $0.3-10 \mathrm{keV}$ band. The SED in Fig. 4 suggests a break between the optical and X-ray bands, with the X-ray part having a steeper slope than its extrapolation to the optical band, in the same sense as observed for OA of GRB 060418 by Molinari et al. (2007). Correction of the $R$ band point by a very $\operatorname{high} A_{R} \approx 3.7$ mag due to the extinction in the host galaxy would make it lie on the extrapolation of the X-ray spectrum in Fig. 4. However, the relation between the optical extinction and X-ray absorption in the host is unknown.

The break in the X-ray light curve occurred in the gap of the optical data, but the ratio of the optical and X-ray flux before and after the break shows that this event is consistent with an achromatic break. Following the approach of Ghirlanda et al. (2004), the Lorentz factor at the time of this break has already decreased to $\Gamma_{\text {break }} \approx 20$.

Although the intrinsic absorbing column of the X-ray afterglow of GRB 090726 is larger than the Galactic one (Page 2009), no changes in its $H$ were observed during its power-law decline covering the part before the break in its light curve (Fig. 3). This 
suggests a constant absorption that does not participate in the evolution of this afterglow. This can be explained if this absorption comes from the regions that are more distant from this GRB, e.g. if this event is behind a molecular cloud. Any possible variations in the absorption caused by emission of this GRB or its afterglow must have occurred before the start of XRT observing, that is at $t-T_{0}<3000 \mathrm{~s}$. The extinction of the optical emission depends on the dust content, while the absorption of X-rays depends on the gaseous component of the absorbing medium. If only the dust is being destroyed during the early phase of the afterglow or condensed during its later phase, then $R X$ would vary, which is not observed in our case (Fig. 3). The constancy of both $R X$ and $H$ (within the observing scatter) suggests that neither the dust nor the gaseous component underwent any evolution during this OA event.

We find that the power-law decay rate of OA of GRB 090726 is comparable to that of the ensemble of OAs with homogeneous optical spectra at $z=0.36-3.5$ (Šimon et al. 2001, 2004). The $k$-corrected absolute magnitude (Šimon et al. 2001) $M_{\mathrm{R}_{0}} \approx-22$ of OA of GRB 090726 at $\left(t-T_{0}\right)_{\text {rest }} \approx 0.2 \mathrm{~d}$ places it below or at the lower end of the distribution of this ensemble of OAs. The $M_{\mathrm{R}_{0}}$ of the ensemble span about 4 mag at this $\left(t-T_{0}\right)_{\text {rest }}$. This is consistent with the synchrotron emission of the GRB afterglow fireball emission model by Sari et al. (1998) with very similar spectra but very different luminosity at a given $\left(t-T_{0}\right)_{\text {rest }}$. In this phase, OA of GRB 090726 thus belongs to the least luminous ones and is similar to OA of X-ray flash XRF 030723 (Fynbo et al. 2004; Šimon et al. 2006).

Acknowledgements. The support by the grants 205/08/1207 and 102/09/0997 of the Grant Agency of the Czech Republic and the projects ESA PECS 98023 and ESA PECS 98058 Gaia is acknowledged. This work made use of data supplied by the UK Swift Science Data Centre at the University of Leicester. We thank Prof. Harmanec for providing us with the program HEC13. The Fortran source version, compiled version and brief instructions how to use the program can be obtained via ftp: //astro. troja.mff. cuni.cz/hec/HEC13.

\section{References}

Akerlof, C., Balsano, R., Barthelmy, S., et al. 1999, Nature, 398, 400 Amati, L., Frontera, F., Tavani, M., et al. 2002, A\&A, 390, 81
Burrows, D. N., Romano, P., Falcone, A., et al. 2005, Science, 309, 1833 Clark, J. S., Miroshnichenko, A. S., Larionov, V. M., et al. 2000, A\&A, 356, 50

Evans, P. A., Beardmore, A. P., Page, K. L., et al. 2007, A\&A, 469, 379 Evans, P. A., Beardmore, A. P., Page, K. L., et al. 2009, MNRAS, 397, 1177 Fatkhullin, T., Gorosabel, J., de Ugarte Postigo, A., et al. 2009, GCN Circ., 9712 Fan, Y.-Z., Piran, T., \& Wei, D.-M. 2008, Astrophysics of compact objects: International Conference on Astrophysics of Compact Objects, AIP Conf. Proc., 968, 32

Fenimore, E. E., Madras, C. D., \& Nayakshin, S. 1996, ApJ, 473, 998 Fynbo, J. P. U., Sollerman, J., Hjorth, J., et al. 2004, ApJ, 609, 962 Ghirlanda, G., Ghisellini, G., \& Lazzati, D. 2004, ApJ, 616, 331 Ghisellini, G., Ghirlanda, G., Nava, L., et al. 2007, ApJ, 658, L75 Greiner, J., Krühler, T., McBreen, S., et al. 2009, ApJ, 693, 1912 Harmanec, P. 1992, ftp://astro.troja.mff.cuni.cz/hec/HEC13 Klotz, A., Boer, M., Atteia, J. L., et al. 2005, A\&A, 439, L35 Kobayashi, S. 2000, ApJ, 545, 807

Kubánek, P., Jelínek, M., Vítek, S., et al. 2006, SPIE, 6274, 59 Lang, D., Hogg, D. W., Mierle, K., et al. 2009, AJ, submitted, [arXiv: 0910.2233]

Maticic, S., \& Skvarc J., 2009, GCN Circ. 9715

Molinari, E., Vergani, S. D., Malesani, D., et al. 2007, A\&A, 469, L13

Monet, D. B. A., Canzian, B., Dahn, C., et al. 1998, VizieR Online Data Catalog, 1252,0

Moskvitin, A., Fatkhullin, T., \& Valeev, A. 2009, GCN Circ., 9709

Nardini, M., Ghisellini, G., Ghirlanda, G., et al. 2006, A\&A, 451, 821

Nysewander, M., Reichart, D. E., Crain, J. A., et al. 2009, ApJ, 693, 1417

Page, K. L. 2009, GCN Circ., 9707

Page, K. L., Ukwatta, T., Cummings, J., et al. 2009, GCN Rep. 234.1

Panaitescu, A., \& Vestrand, W. T. 2008, MNRAS, 387, 497

Rykoff, E. S., Aharonian, F., Akerlof, C. W., et al. 2009, ApJ, 702, 489

Sari, R., Piran, T., \& Narayan, R. 1998, ApJ, 497, L17

Schlegel, D. J., Finkbeiner, D. P., \& Davis, M. 1998, ApJ, 500, 525

Šimon, V., Hudec, R., Pizzichini, G., et al. 2001, A\&A, 377, 450

Šimon, V., Hudec, R., Pizzichini, G., et al. 2004, Gamma-Ray Bursts: 30 Years of Discovery: Gamma-Ray Burst Symposium, ed. E. E. Fenimore, \& M. Galassi, Melville (NY: AIP), AIP Conf. Proc., 727487

Šimon, V., Hudec, R., \& Pizzichini, G. 2006, Il Nuovo Cimento C, Proceedings of Swift and GRBs: Unveiling the Relativistic Universe, $121 \mathrm{~B}, 1579$

Volnova, A., Pavlenko, E., Sklyanov, A., et al. 2009, GCN Circ., 9741

Vondrák, J. 1969, Bull. Astron. Inst. Czechosl., 20, 349

Vondrák, J. 1977, Bull. Astron. Inst. Czechosl., 28, 84

Whittaker, E., \& Robinson, G. 1946, The Calculus of Observations (Blackie \& Son Ltd, London), 303

Willingale, R., O’Brien, P. T., Osborne, J. P., et al. 2007, ApJ, 662, 1093

Zhang, B., \& Kobayashi, S. 2005, ApJ, 628, 315

Zhang, B., Kobayashi, S., \& Meszaros, P. 2003, ApJ, 595, 950 